Case Report

\title{
Aggressive Disease Course of Multiple Myeloma with Concomitant ALK-Negative Anaplastic Large Cell Lymphoma: A Case Report with an Unusual Presentation
}

\author{
Michela Staderini (D), ${ }^{1}$ Lara Mannelli, ${ }^{1,2}$ Elisabetta Antonioli, ${ }^{1}$ Benedetta Puccini, \\ Valentina Berti, ${ }^{3}$ Francesco Mungai, ${ }^{4}$ Federica Vergoni, ${ }^{5}$ Valentina Carrai, ${ }^{1}$ \\ Luigi Rigacci, ${ }^{1,6}$ and Alberto Bosi ${ }^{1}$ \\ ${ }^{1}$ Haematology Unit, Careggi University Hospital, Florence, Italy \\ ${ }^{2}$ Medical Genetics, University of Siena, Siena, Italy \\ ${ }^{3}$ Nuclear Medicine Unit, Department of Biomedical, Experimental and Clinical Sciences, Careggi University Hospital, \\ Florence, Italy \\ ${ }^{4}$ Diagnostic Radiology Unit, Careggi University Hospital, Florence, Italy \\ ${ }^{5}$ Pathological Anatomy Unit, Careggi University Hospital, Florence, Italy \\ ${ }^{6}$ Hematology and Stem Cell Transplant Unit, San Camillo Forlanini Hospital, Rome, Italy \\ Correspondence should be addressed to Michela Staderini; michelastaderini@gmail.com
}

Received 11 June 2019; Revised 9 December 2019; Accepted 28 December 2019; Published 16 January 2020

Academic Editor: Yusuke Shiozawa

Copyright (c) 2020 Michela Staderini et al. This is an open access article distributed under the Creative Commons Attribution License, which permits unrestricted use, distribution, and reproduction in any medium, provided the original work is properly cited.

\begin{abstract}
ALK-negative anaplastic large cell lymphoma is a rare T-cell neoplasm with an aggressive course requiring prompt diagnostic work-up and treatment. Few cases of concomitant multiple myeloma and T-cell neoplasm are described in the literature, mainly regarding primary cutaneous anaplastic large cell lymphoma. We present the case of a 65 -year-old man, simultaneously diagnosed with ALK-negative anaplastic large cell lymphoma with extranodal localization in the gastrocnemius muscle (stage $1 \mathrm{AE}$ ) and IgG lambda multiple myeloma (ISS 2, Durie-Salmon stage 3A). Both diseases required therapeutic intervention due to the high proliferative index of lymphoma and the presence of bone lesions attributable to myeloma. The therapeutic program initially included chemotherapy (cyclophosphamide, doxorubicin, vincristine, etoposide, and prednisone; CHOEP), radiotherapy on the leg, bortezomib, and then consolidation with autologous hematopoietic stem cell transplantation. Despite being on bortezomib treatment and waiting for transplantation, the patient experienced an early myeloma progression that turned out to be refractory to second-line lenalidomide-based treatment. To our knowledge, this is the first case of concurrent diagnosis of extranodal ALKnegative anaplastic large cell lymphoma of the muscle and multiple myeloma. Simultaneous onset can be challenging for clinicians as both diseases may have an aggressive course requiring multiple treatments with increased risk of toxicity and complicated management.
\end{abstract}

\section{Introduction}

ALK-negative anaplastic large cell lymphoma (ALK-ALCL) is a rare CD30+ T-cell neoplasm involving both lymph nodes and extranodal sites, often presenting with B symptoms and advanced stage disease [1]. Primary involvement of the skeletal muscles is extremely rare in adults and has been associated with rapid dissemination and poor prognosis [2-4].

On the other side, multiple myeloma (MM) is characterized by clonal plasma cell (PC) infiltration in the bone marrow and, rarely, in extramedullary sites, leading to signs of organ damage typically summarized by the acronym 
CRAB (hypercalcemia, renal failure, anaemia, and bone lesions).

Few cases of concomitant diagnosis of multiple myeloma and T-cell neoplasms are described, mainly regarding CD30+ cutaneous anaplastic large cell lymphoma. Often the plasma cell dyscrasia precedes the diagnosis of the T-cell neoplasm [5-7]. Some authors have hypothesized a causal relationship between the two diseases, but the potential pathogenic mechanism has not been fully understood [7]. Currently, only one case of contemporary diagnosis of aggressive ALK-negative ALCL and IgA lambda multiple myeloma is described in the literature [8]; that case was characterized by $15 \%$ plasma cell infiltration in the bone marrow (suggestive of smoldering MM), and no information on follow-up or response to treatment was available because the patient died quickly after diagnosis.

To the best of our knowledge, this is the first report of a simultaneous diagnosis of symptomatic extranodal ALKALCL and MM, requiring to start a treatment aiming to target both diseases.

\section{Case Presentation}

In June 2017, a Caucasian 65-year-old man was admitted to the Hematology Unit of Careggi Hospital (Florence, Italy) with swelling and pain in his right leg. At MRI, a large spindle-shaped lesion (size $3 \times 2.2 \times 8.4 \mathrm{~cm}$ ) was detected at his right gastrocnemius muscle. The lesion had the same signal intensity of the surrounding normal muscular tissue in the T1-weighted sequence and intermediate signal intensity in the T2-weighted sequence; it was hyperintense in sequences with fat suppression and showed a high contrastenhancement.

$\mathrm{He}$ underwent a core needle biopsy of the lesion with morphologic examination demonstrating a dense infiltrate of large pleomorphic cells with prominent nucleoli, many of which having horseshoe or kidney-shaped eccentric nuclei resembling hallmark cells (Figure 1). Immunohistochemical staining showed expression of CD45 and CD4, strong and uniform expression of CD30, and negativity of CD19, CD20, Pax-5, CD3, CD5, CD8, EMA, Perforin, CD56, CD34, and ALK in the neoplastic cells; TIA1 was only expressed in a minority of cells $(-/+)$; MIB-1 was $>90 \%$. These findings were consistent with ALK-negative anaplastic large cell lymphoma (Figure 2).

The patient did not complain about any systemic symptom; his physical exam was normal. He had no previous medical history of cancer or autoimmune diseases. In his medical records, atrial fibrillation and ventricular flutter on treatment with beta-blockers were reported. Blood tests showed normal white blood cell and platelet counts, with mild anaemia (haemoglobin $12.5 \mathrm{~g} / \mathrm{d}$ ); $\mathrm{LDH}$ and liver enzymes were normal, creatinine $1.25 \mathrm{mg} / \mathrm{dl}$, calculated GFR $54 \mathrm{ml} / \mathrm{min}$, and beta 2 microglobulin $4.5 \mathrm{mg} / \mathrm{L}$. In addition, a serum IgG lambda monoclonal component $(1.53 \mathrm{~g} / \mathrm{dl})$ was found, with increased lambda light chains $(106 \mathrm{mg} / \mathrm{L})$, and the sFLC ratio was lower than 100 , albumin $33.7 \mathrm{~g} / \mathrm{L}$, and calcium $8.9 \mathrm{mg} / \mathrm{dl}$.

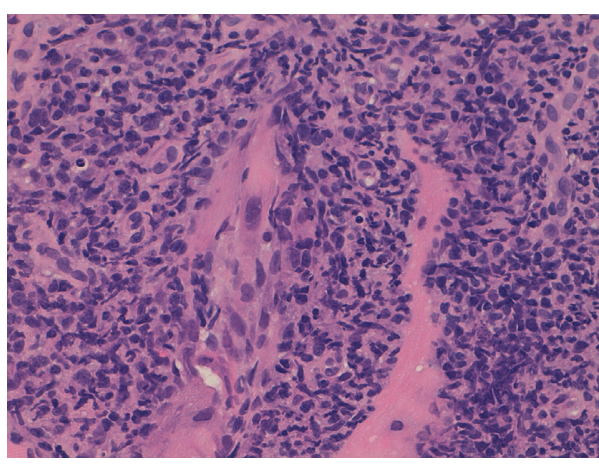

Figure 1: Core needle biopsy of the lesion in the right gastrocnemius muscle. H\&E stain showing a dense infiltrate of large pleomorphic cells with prominent nucleoli and horseshoe or kidney-shaped nuclei (hallmark cells) consistent with ALCL.

A whole-body computed tomography (CT) scan did not show any other pathologic finding; in particular, no bone lytic lesions were revealed. In order to complete lymphoma staging procedures, a bone marrow biopsy was performed, and a relevant infiltration (roughly $70 \%$ ) by atypical plasma cells with lambda light-chain restriction was detected (Figure 3).

Multiparameter flow cytometry on the bone marrow sample showed plasma cells, accounting for $45 \%$ of cellularity, that were positive for $\mathrm{CD} 138$ and $\mathrm{CD} 38$ and negative for CD19, CD56, and CD45; CD117 was partially expressed. No signs of bone marrow clonal T-cell infiltration were found. Cytogenetic analysis was not performed because of insufficient bone marrow sample.

An F18-FDG PET/CT confirmed the lesion in the right gastrocnemius muscle, which was intensely hypermetabolic, and revealed three additional vertebral hypermetabolic lesions in D5, L2, and S1, that were ascribed to myeloma (Figure 4(a)).

Based on these findings, the patient was diagnosed with an aggressive CD30+ ALK-negative ALCL (stage 1AE) and a concomitant IgG lambda multiple myeloma (MM, ISS score 2, and Durie-Salmon stage IIIA). Due to one MM-defining event (bone lesions at F18-FDG PET/CT) and one biomarker of malignancy (bone marrow infiltration over 60\%), myeloma was defined as symptomatic, thus indicating the need to start an effective treatment.

The concurrence of two aggressive diseases led us to adopt a treatment approach taking into account the high proliferative index of lymphoma and therefore was based on the immediate application of standard ALCL treatment, followed by a consolidation program including autologous stem cell transplantation (ASCT), preceded by a conditioning regimen also active on myeloma.

From August 2017 to November 2017, the patient received 4 courses of polychemotherapy according to the CHOEP regimen (21-day cycles of cyclophosphamide $750 \mathrm{mg} / \mathrm{sqm}$, doxorubicin $50 \mathrm{mg} / \mathrm{sqm}$, and vincristine $1.4 \mathrm{mg} / \mathrm{sqm}$ on day 1 ; etoposide $100 \mathrm{mg} / \mathrm{sqm}$ on days $1-3$; prednisone $100 \mathrm{mg} / \mathrm{sqm}$ on days $1-5$ ).

In January 2018, after chemotherapy, an MRI showed a complete response of the ALCL lesion, and a 18F-FDG PET/ 


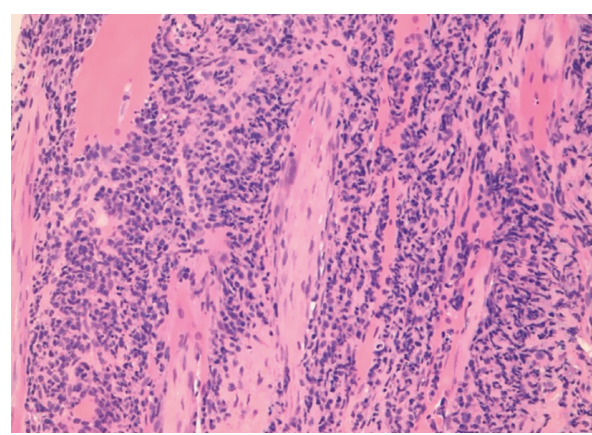

(a)

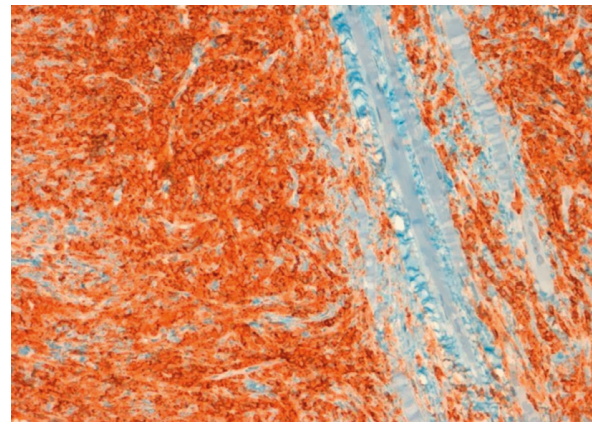

(c)

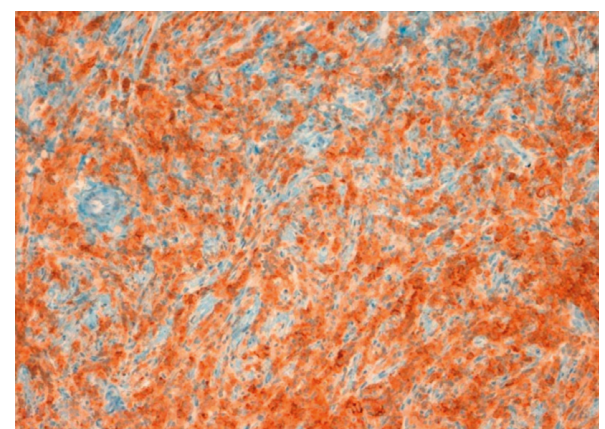

(b)

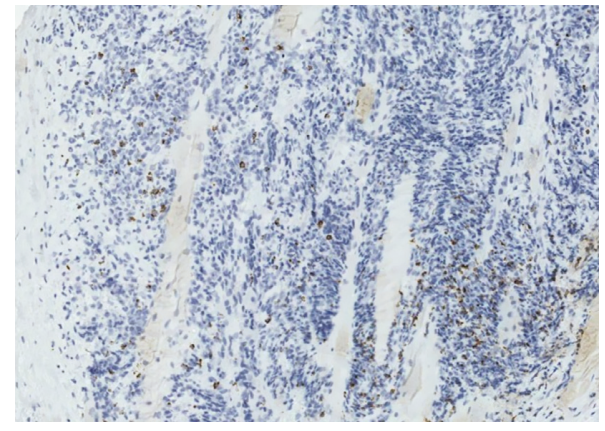

(d)

Figure 2: Immunohistochemical features of the lymphoma. (a) Hematoxylin and eosin staining; (b) CD30 and (c) CD4 immunostaining showing a strong and uniform expression in the neoplastic cells; (d) TIA1 immunostaining; this marker was only expressed in a minority of cells $(-/+)$.

CT revealed the complete disappearance of the right gastrocnemius muscle and of D5 and L2 lesions, with persistence of moderate metabolic activity in the left sacral lesion (Deauville score $=3$ ) (Figure 4(b)). Serum M-component decreased $(0.4 \mathrm{gr} / \mathrm{dl})$, and a re-evaluation of bone marrow biopsy displayed the reduction of plasma cell infiltration to $10 \%$ of marrow cellularity. As such, MM was in partial response. On February 2018, peripheral blood stem cell (PBSC) mobilization was achieved following high-dose cyclophosphamide ( $2 \mathrm{gr} /$ sqm) and granulocyte-colony stimulating factor (G-CSF), and an amount of CD34+ cells adequate for a single ASCT was collected. Then, in March 2018, the patient received localized radiotherapy on the right gastrocnemius muscle for a total of $36 \mathrm{~Gy}$, without any relevant complication. Waiting to be transplanted, on April 2018, the patient started VD treatment (bortezomib $1.3 \mathrm{mg} / \mathrm{smq}$ twice a week, days 1-4-8-11 and oral dexamethasone $40 \mathrm{mg}$, days 1-2-4-5-8-9-11-12, cycles every 21 days), completing two cycles. On June 2018, during the third course, he was hospitalized because of an E. coli infection complicated by sepsis and congestive heart failure; he was discharged one month later. Serum M-protein remained permanently around $0.3 \mathrm{~g} / \mathrm{dl}$, and serum lambda light chains were $30 \mathrm{mg} / \mathrm{L}$, confirming a $\mathrm{MM}$ partial response.

Unfortunately, on July 2018, few days later, he was hospitalized again due to paraplegia and neurogenic bladder dysfunction. An MRI of the spine showed a spinal cord compression due to neoplastic tissue extending from vertebrae D6 to D10 (size $10 \times 5 \times 2.5 \mathrm{~cm}$ ). Considering the severity of the neurologic impairment, a new biopsy of the lesion was not performed, and radiotherapy in association to high-dose dexamethasone was immediately started, obtaining a rapid neurologic improvement.

M-protein kept stable $(0.3 \mathrm{~g} / \mathrm{dl})$, but lambda sFLC were increased to $860 \mathrm{mg} / \mathrm{L}$. A new bone marrow biopsy showed an increased monoclonal plasma cell infiltration $(50 \%$ of marrow cellularity) with morphologic atypia and aberrant phenotype. Fluorescent in situ hybridization (FISH) performed on purified bone marrow plasma cells, which was not available at the diagnosis, showed $t(14 ; 16)$ in $100 \%$ and del17p13 in 64\% of the cells analyzed. On August 2018, we started a second line therapy with weekly daratumumab in combination with lenalidomide and dexamethasone (DRd). After one cycle, the patient's conditions deteriorated, and a new MRI of the spine revealed two additional neoplastic lesions that extended from vertebrae D2 to D6 and from vertebrae D12 to L4. In September 2018, the patient was deceased due to disease progression.

\section{Discussion}

ALK- ALCL usually presents with advanced stage disease (stage III-IV), B symptoms, lymph nodes, and extranodal tissue involvement [1]. The median age at presentation of ALK- ALCL patients is 54-61 years [9].

Our case instead presented with an extranodal noncutaneous ALK- ALCL showing muscular involvement as a unique lesion without systemic symptoms. This clinical onset coincided with a symptomatic IgG lambda MM. According to the last edition of WHO classification, ALKALCL is defined as a CD30+ T-cell neoplasm that is not 


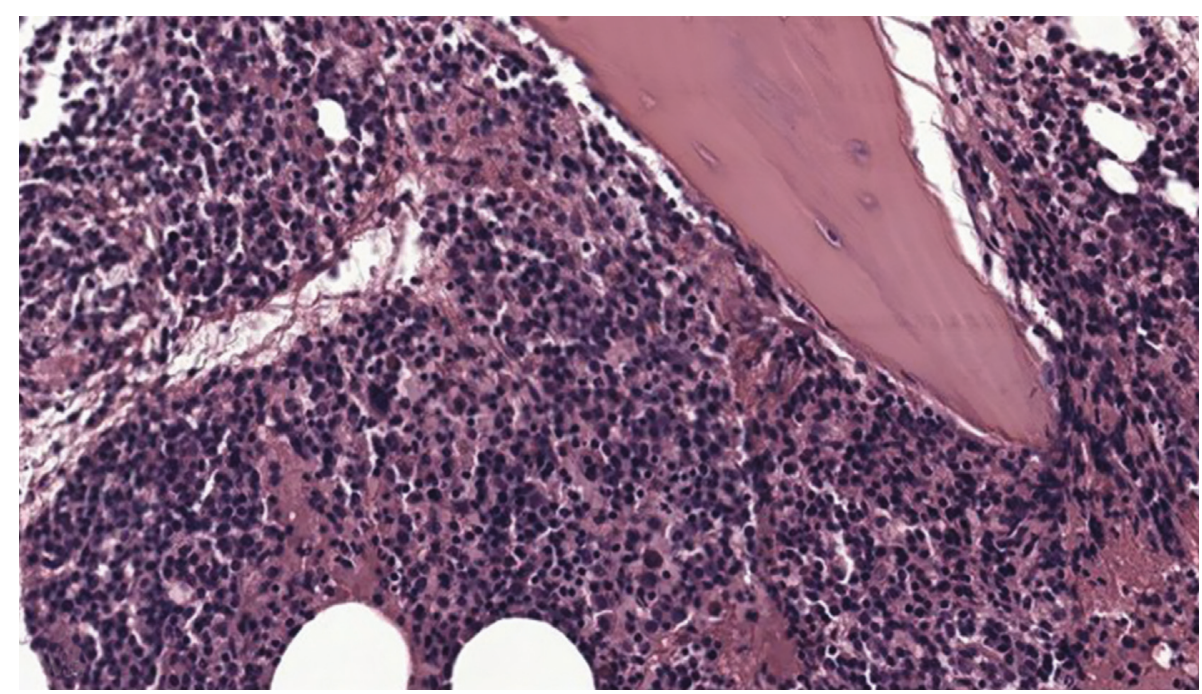

(a)

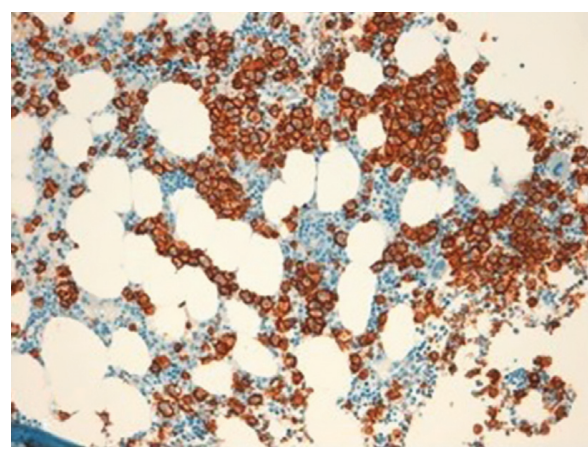

(b)

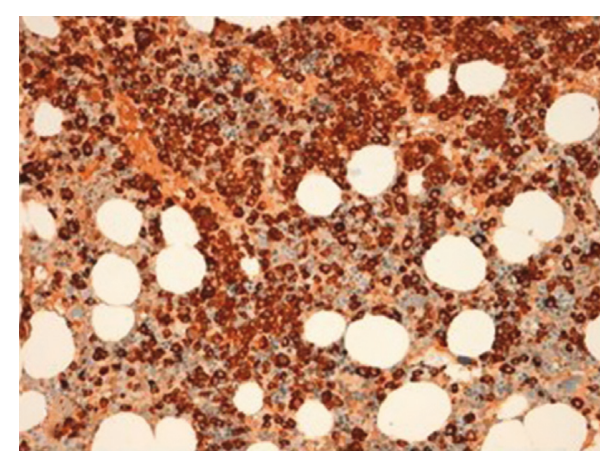

(c)

FIGURe 3: Bone marrow biopsy performed at diagnosis showed plasma cell infiltration with lambda-chain restriction. (a) H\&E staining; (b) CD138 staining; (c) lambda staining.

reproducibly distinguishable by morphology from ALKpositive (ALK+) ALCL, but lacks ALK protein expression [1]. T-cell/cytotoxic markers can be lacking in ALCL as well as EMA which is only positive in $43 \%$ of the cases [10]. While both Pax-5 negativity and cell morphology helped us to rule out classical Hodgkin lymphoma, distinguishing ALCL from PTCL-NOS can be challenging. The occurrence of hallmark cells together with strong and uniform CD30 expression in the neoplastic proliferation appeared more consistent with ALCL than PTCL-NOS in this case.

Regarding concomitant diagnosis of T-cell lymphoma and MM, in the few other cases described in the literature [5-7], the diagnosis of a T-cell neoplasm occurred later than the diagnosis of $\mathrm{MM}$, leading to surmise a pathogenic role for acquired cellular immunity impairment, on turn caused by PC disorders or previous therapies. In our patient the relationship between the two neoplasms was unclear, and the chronologic sequence of onset could not be proven. Nevertheless, the patient did not have any prior history of MGUS or other plasma cell dyscrasias. Moreover, the incidence of lymphoproliferative disorders is generally increased in immunosuppressed patients, but our patient was not previously treated with any chemo-radiotherapy and blood tests excluded an active viral infection.
The optimal treatment for ALK-negative ALCL is still debated because of the rarity of the disease and the related lack of prospective randomized trials. ALK- ALCL generally responds well to doxorubicin-containing chemotherapy, but frequently relapses [11]. The role of autologous stem cell transplant in first remission in ALCL has been investigated in small trials with 5-year overall survival (OS) rates of up to $80 \%$. However, in many of these studies, ALK expression was not assessed $[12,13]$. The prognosis of ALK- ALCL patients is poor, with a 5 -year (OS) of $30-49 \%$, versus $70-86 \%$ in ALK+ ALCL $[14,15]$. On the contrary, multiple myeloma treatment is well standardized and includes upfront autologous stem cell transplantation for younger patients [16]. However, the prognosis can be quite heterogeneous according to cytogenetic and biochemical markers [17]: specifically, the outcome of patients harbouring one of the high-risk chromosomal aberrations, such as $t(4 ; 14), t(14 ; 16)$, $t(14 ; 20)$, and especially del17p, is dismal and not completely overcome by the new drug combinations so far [18].

In our patient, both diseases required therapeutic intervention. Because of the high proliferation index (MIB$1>90 \%$ ), we decided to treat lymphoma first. Afterwards, we observed an aggressive behaviour and a rapid progression of multiple myeloma which led the patient to death, eventually 


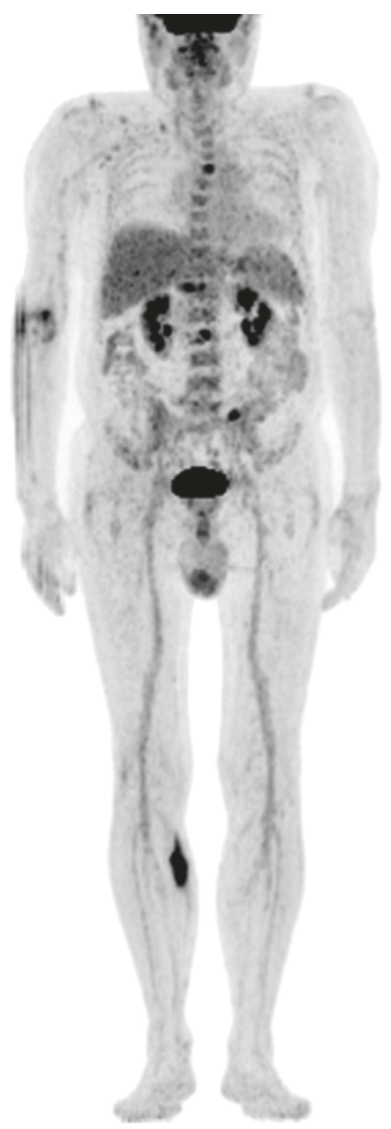

(a)

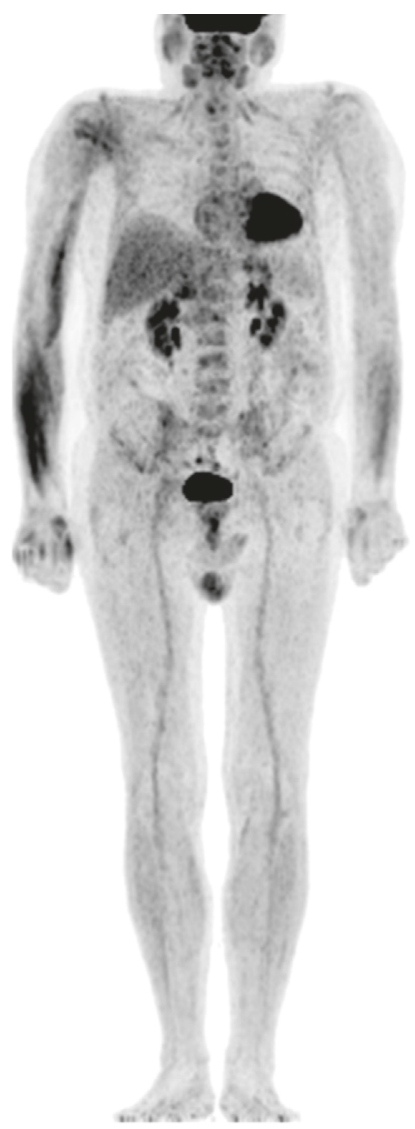

(b)

FIGURE 4: F18-FDG PET/CT performed at staging showed an intensely hypermetabolic lesion in the right leg and revealed three additional vertebral hypermetabolic lesions in D5, L2, and S1 (a). After chemotherapy, the hypermetabolic lesions in the right leg, D5, and L2 had completely disappeared, with persistence of moderate metabolic activity in the left sacral lesion (DS=3) (b).

explained by the double high-risk cytogenetic alterations identified at relapse.

Unfortunately, no cytogenetic data were available at diagnosis, and we cannot exclude a clonal selection secondary to chemotherapy treatment for lymphoma.

\section{Ethical Approval}

This retrospective study was approved by our institutional board and by our ethical committee (Comitato Etico Area Vasta Centro) and has been performed in accordance with the ethical standards of the Declaration of Helsinki.

\section{Consent}

Written informed consent was obtained from the patient's relatives for publication of this case report and any accompanying images.

\section{Conflicts of Interest}

The authors declare that there are no conflicts of interest regarding the publication of this article.

\section{Authors' Contributions}

Michela Staderini and Lara Mannelli are contributed equally to the manuscript.

\section{References}

[1] S. H. Swerdlow, E. Campo, S. A. Pileri et al., "The 2016 revision of the World Health Organization classification of lymphoid neoplasms," Blood, vol. 127, no. 20, pp. 2375-2390, 2016.

[2] M. Emori, M. Kaya, S. Takahata, H. Tobioka, Y. Minaki, and T. Yamashita, "Anaplastic lymphoma kinase-negative anaplastic large cell lymphoma with extranodal involvement of the thigh muscle: a case report," Journal of Medical Case Reports, vol. 8, no. 1, p. 9, 2014.

[3] Y. Kubo, J. Aoi, T. Johno et al., "A case of anaplastic large cell lymphoma of skeletal muscle," The Journal of Dermatology, vol. 41, no. 41, pp. 999-1002, 2014.

[4] Y. Zongyou, L. Yueju, G. Fuqian et al., "Anaplastic large cell lymphoma with primary involvement of the skeletal muscle: a case report," Oncology Letters, vol. 9, no. 6, pp. 2815-2818, 2015.

[5] R. C. Romano, D. N. Cohen, M. T. Howard, and C. N. Wieland, "A primary cutaneous CD30-positive T-cell lymphoproliferative disorder arising in a patient with multiple 
myeloma and cutaneous amyloidosis," The American Journal of Dermatopathology, vol. 38, no. 5, pp. 388-392, 2016.

[6] M. Nassiri, G. E. Byrne, C. C. Whitcomb, and J. J. Byrnes, "Synchronous null-cell anaplastic large cell lymphoma and multiple myeloma," Annals of Hematology, vol. 88, no. 9, pp. 923-925, 2009.

[7] M. Tangour, I. Chelly, S. Haouet, M. Zitouna, and N. Kchir, "Multiple myeloma and cutaneous anaplastic large T-cell lymphoma in the same patient: is there a causal relation?," Journal of Cutaneous Pathology, vol. 38, no. 3, pp. 298-300, 2011.

[8] S. Nassif, N. El-Majzoub, O. Abbas, S. Temraz, and Z. Chakhachiro, "Simultaneous occurrence of a CD30 positive/ALK-negative high grade T-cell lymphoma and plasma cell myeloma: report of a case," Hematology/Oncology and Stem Cell Therapy, vol. 8, no. 1, pp. 40-42, 2015.

[9] C. Querfeld, I. Khan, B. Mahon, B. P. Nelson, S. T. Rosen, and A. M. Evens, "Primary cutaneous and systemic anaplastic large cell lymphoma: clinicopathologic aspects and therapeutic options," Oncology (Williston Park), vol. 24, no. 7, pp. 574-587, 2010.

[10] K. J. Savage, N. L. Harris, J. M. Vose et al., "ALK- anaplastic large-cell lymphoma is clinically and immunophenotypically different from both ALK+ ALCL and peripheral T-cell lymphoma, not otherwise specified: report from the International Peripheral T-Cell Lymphoma Project," Blood, vol. 111, no. 12, pp. 5496-5504, 2008.

[11] A. J. M. Ferreri, S. Govi, S. A. Pileri, and K. J. Savage, "Anaplastic large cell lymphoma, ALK-negative," Critical Reviews in Oncology/Hematology, vol. 85, no. 2, pp. 206-215, 2013.

[12] R. Fanin, F. Silvestri, A. Geromin et al., "Primary systemic CD30 (Ki-1)-positive anaplastic large cell lymphoma of the adult: sequential intensive treatment with the F-MACHOP regimen (+/- radiotherapy) and autologous bone marrow transplantation," Blood, vol. 87, no. 4, pp. 1243-1248, 1996.

[13] R. Fanin, A. Sperotto, F. Silvestri et al., "The therapy of primary adult systemic CD30-positive anaplastic large cell lymphoma: results of 40 cases treated in a single center," Leukemia \& Lymphoma, vol. 35, no. 1-2, pp. 159-169, 1999.

[14] K. J. Savage, "Prognosis and primary therapy in peripheral T-cell lymphomas," Hematology, vol. 2008, no. 1, pp. 280-288, 2008.

[15] H. Stein, H. D. Foss, H. Dürkop et al., "CD30(+) anaplastic large cell lymphoma: a review of its histopathologic, genetic, and clinical features," Blood, vol. 96, no. 12, pp. 3681-3695, 2000.

[16] F. Gay, M. Engelhardt, E. Terpos et al., "From transplant to novel cellular therapies in multiple myeloma: European Myeloma Network guidelines and future perspectives," Haematologica, vol. 103, no. 2, pp. 197-211, 2018.

[17] A. Palumbo, H. Avet-Loiseau, S. Oliva et al., "Revised International Staging System for multiple myeloma: a report from International Myeloma Working Group," Journal of Clinical Oncology, vol. 33, no. 26, pp. 2863-2869, 2015.

[18] P. Sonneveld, H. Avet-Loiseau, S. Lonial et al., "Treatment of multiple myeloma with high-risk cytogenetics: a consensus of the International Myeloma Working Group," Blood, vol. 127, no. 24, pp. 2955-2962, 2016. 


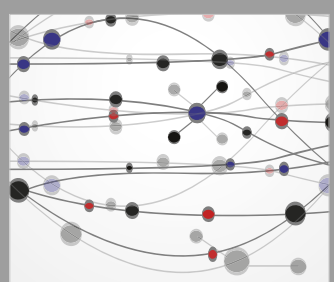

The Scientific World Journal
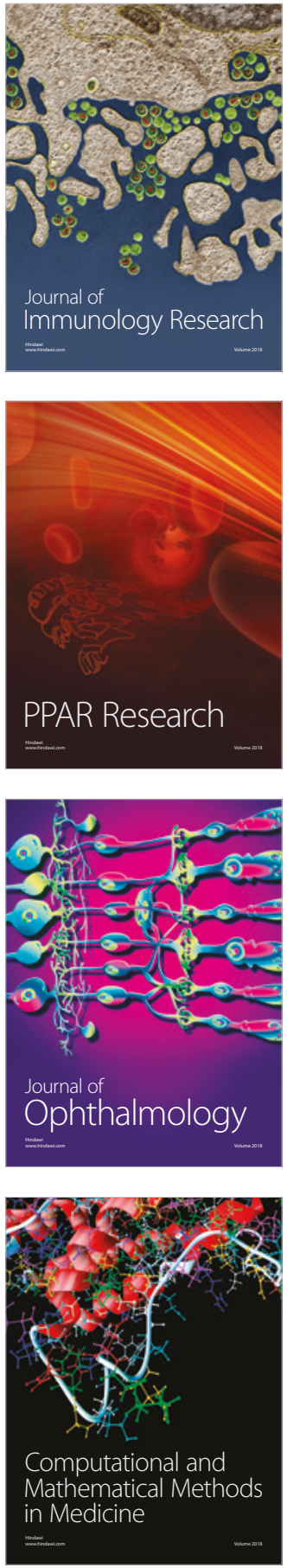

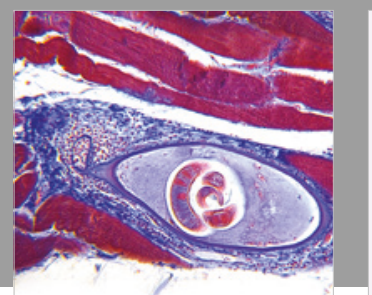

Gastroenterology Research and Practice

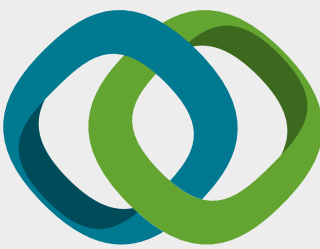

\section{Hindawi}

Submit your manuscripts at

www.hindawi.com
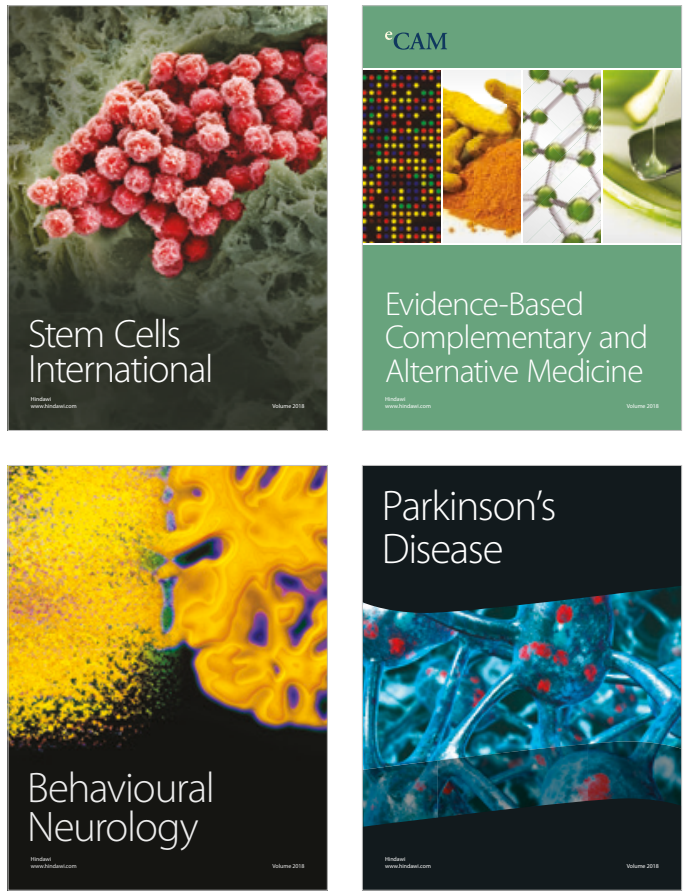

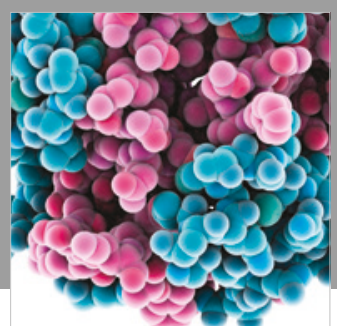

ournal of

Diabetes Research

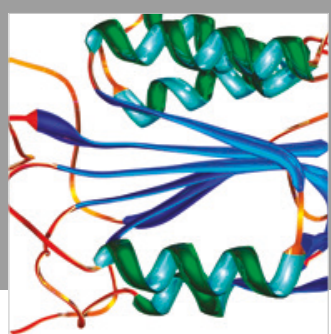

Disease Markers
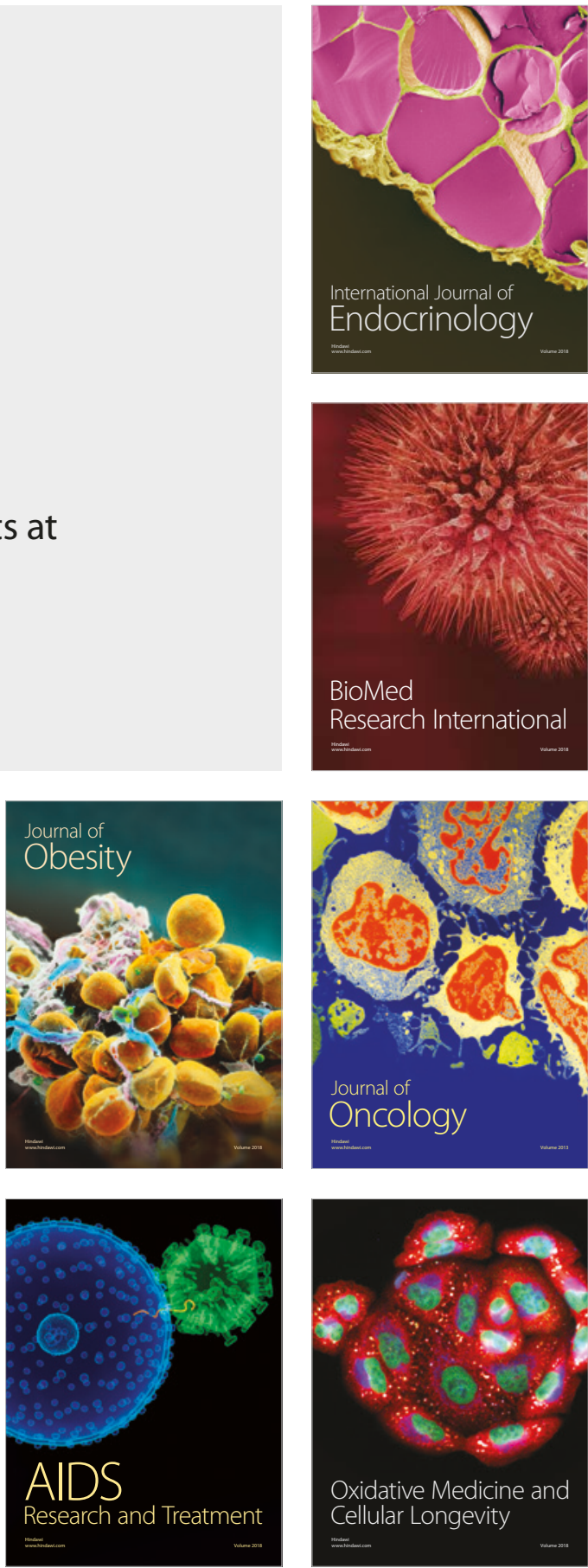